\title{
Gefühle und Gedanken teilen
}

\author{
Mindsharing in der Gruppe Gedanken und Gefühle äußern. Zuhören. Sich austauschen.
}

Das sogenannte Mindsharing ermöglicht es, sich in Krisen zum Beispiel auch per Video

psychisch zu stabilisieren und Nähe zu erfahren.

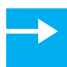

Die globale Corona-Krise ist eine von Angst, Unsicherheit und Einsamkeit geprägte Zeit. Diese schlagartig veränderte, befremdliche Realität erfordert eine neue Herangehensweise, um die eigenen Gefühle und Gedanken zu sortieren. Die Pandemie bringt laut Karen Whalley Hammell eine „occupational disruption" mit sich [1]. Damit ist ein Abbruch oder eine Unterbrechung der täglichen Routine und der gewohnten, regelmäßig ausgeführten Betätigungen gemeint. Diese Unterbrechung lag außerhalb unserer Kontrolle. Sie war der Ausbreitung von COVID-19 und der darauf reagierenden politischen Entscheidungen geschuldet.

Das Leben davor schien weitestgehend berechenbar - ein Kalender voller Termine, ein Alltag geprägt von Verpflichtungen, Ortswechseln und Events. Mit der Pandemie fielen sämtliche Betätigungsmöglichkeiten in der Bildungsund Arbeitswelt, auf öffentlichen Plätzen, in Gebäuden sowie kulturelle Angebote und soziale Begegnungen abrupt weg [1]. Eine plötzlich fehlende Struktur kann ein Gefühl von Ungewissheit auslösen und zu starker psychischer Belastung führen. Deshalb empfiehlt der Psychiater Manfred Spitzer (2020) während einer erzwungenen Auszeit, in sich zu gehen und über sich und das Leben zu reflektieren.

Diesen Rat nahmen wir im Studiengang Ergotherapie an der Fachhochschule Wiener Neustadt, Österreich, an. Wir nutzten dafür das Mindsharing, zu Deutsch „Gedanken teilen“, in der Gruppe. Damit war ein psychosoziales Gesundheitsförderungsprojekt des Bachelorabschlussjahrgangs geboren. Die Idee der bewussten Wahrnehmung und des Teilens von Gedanken und Emotionen entstand im März 2020, als die Corona-Krise gerade begann, ihren Lauf zu nehmen.

\section{Gedankenaustausch in zwei Runden $\rightarrow$ Die} eigene Meinung und Befindlichkeit offen und intuitiv zu teilen, lässt Gemeinschaft entstehen. Die anthropologischen Ursprünge dieser Intervention entstammen den „talking circles“ der nordamerikanischen Ureinwohner/-innen [2]. Dieses offene System des Teilens von Gedanken und Seinszuständen wurde ab den 1970er Jahren von nicht indigenen kulturellen Gegenbewegungen übernommen und damit anderen sozialen Gruppen zugänglich gemacht [3]. Wir vermuten, dass dieses System von England ausgehend in unterschiedlichen Workshops mit psychodynamischem Schwerpunkt in Europa und Nordamerika weiterentwickelt wurde. Die „talking circles“ wurden dadurch an den Zeitgeist westlicher Gesellschaften angepasst. Die modifizierte Form besteht aus zwei Teilen:

$\rightarrow$ In der ersten Runde erhalten alle Teilnehmenden die Möglichkeit, zeitlich begrenzt ihre Gedanken und Gefühle zu äußern.

$\rightarrow$ In der zweiten Gesprächsrunde nehmen sie nacheinander Bezug auf eine konkrete Frage. Diese Form setzten wir im Lockdown online um und leisten damit nach unserem Kenntnisstand Pionierarbeit.

Occupational Disruption $\rightarrow$ Körperliche sowie psychische Gesundheitsförderung und Prävention sind Aufgabenbereiche der Ergotherapie [4]. Die Corona-Krise führt laut Torales et al. (2020) weltweit zu einem vermehrten Auftreten von Stress, Sorge, depressiver Verstimmung, Schlafproblemen, Ablehnung, Wut und Angst [9]. Daraus resultiert aus unserer Sicht aktuell ein erhöhter Bedarf für psychische Gesundheitsförderung durch Ergotherapie.

Die Pandemie geht mit einer „occupational disruption“ einher, also mit einer Störung oder

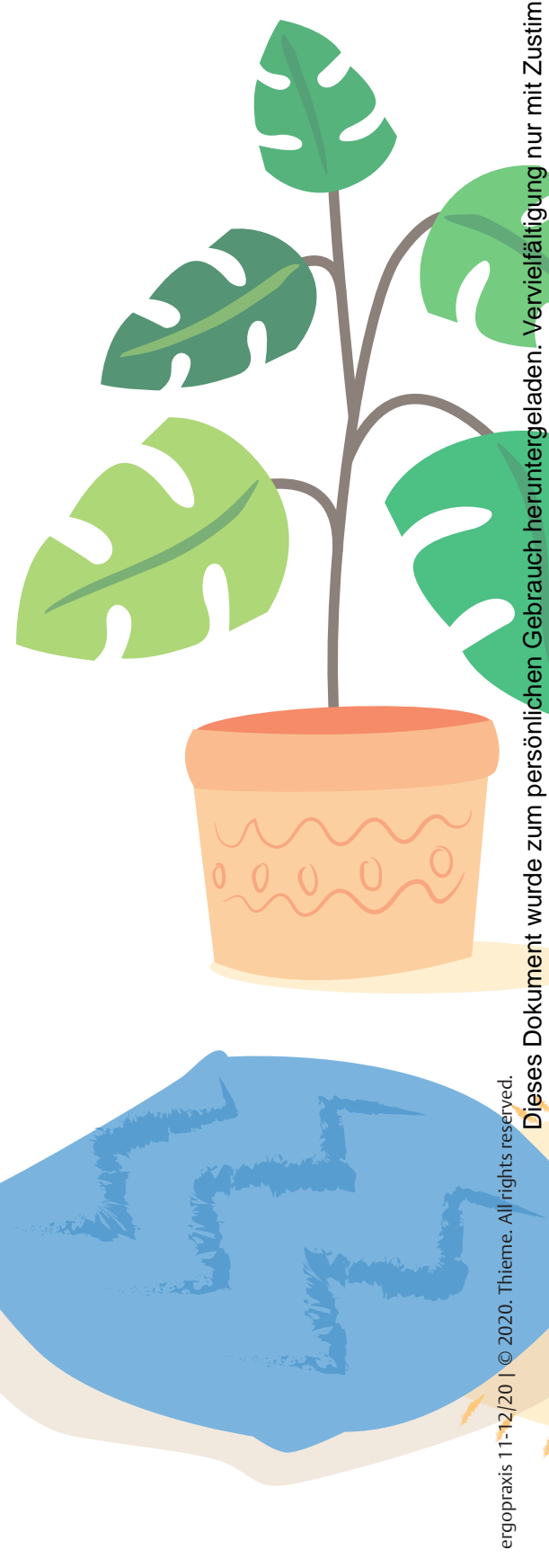




\section{9}

Mindsharing war für uns eine Möglichkeit, regelmäßig online darüber zu sprechen, was uns gerade beschäftigt. Zudem

lernten wir, die Emotionen anderer zu hören, ohne eine Lösung dafür suchen zu müssen. Zusammenfassend brachte uns die Teilnahme an der Gruppe Inspiration, Freude und sozialen Kontakt trotz Isolation.

Das Programm und die Gruppe finde ich sehr bereichernd, da der Austausch toll ist, man sich im aktiven Zuhören üben

kann und selbst gefordert ist, anderen zu erzählen. Mindsharing bietet für mich nur Benefits, vom gegenseitigen Austausch über den Einblick in die Gedanken von anderen bis hin zu der Möglichkeit, eigene Gedanken und Erlebnisse zu teilen.

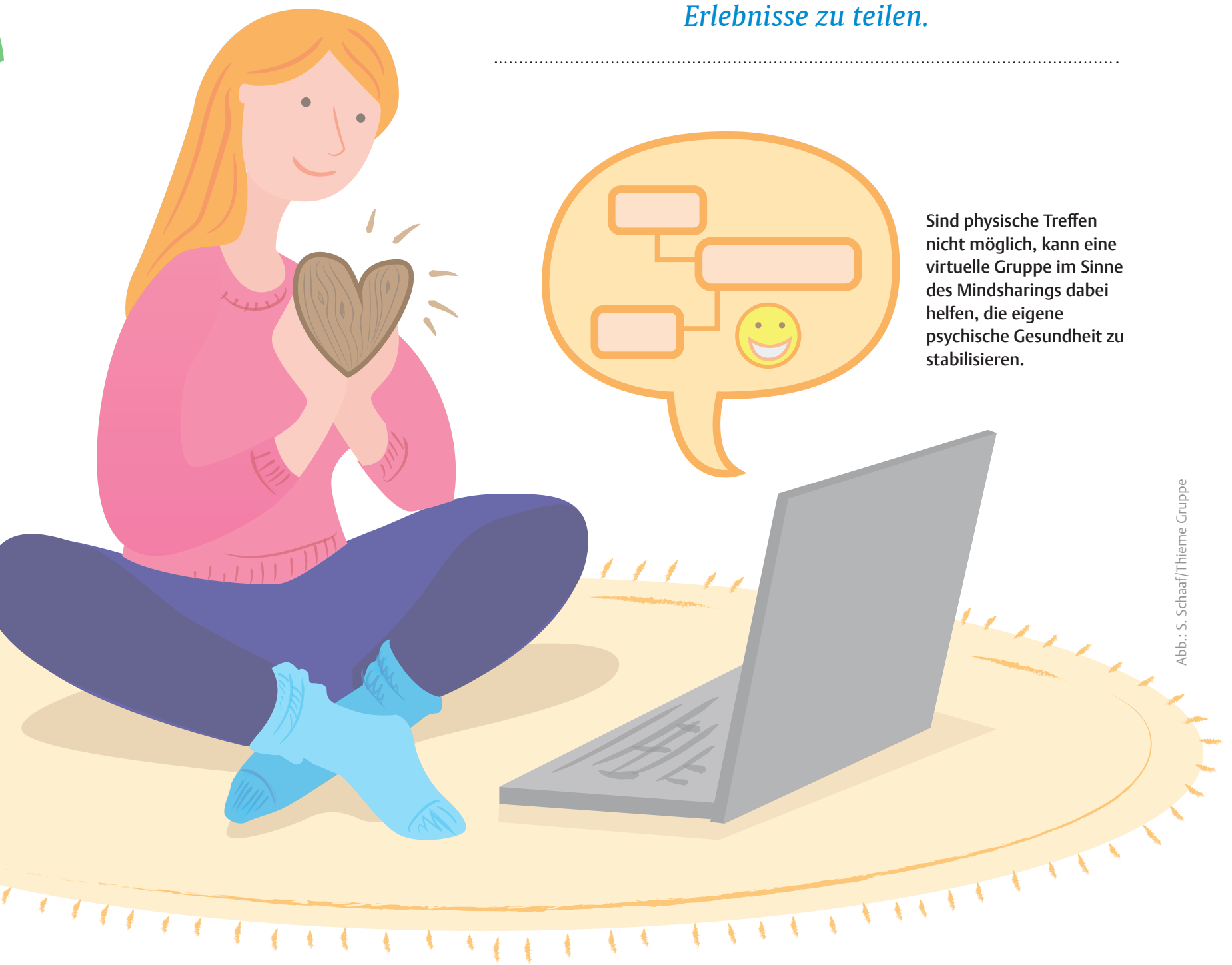




\section{$\rightarrow$ Beispiel}

\section{Einblick in einen Gruppentermin}

Heute leite ich zum ersten Mal eine unserer Mindsharing-Gruppen. Meine Aufgaben sind das Stoppen der Zeit, das Anleiten der Zentrierungsübung und die Formulierung der Frage für die zweite Gesprächsrunde. Ansonsten nehme auch ich ganz normal an der Runde teil. Klingt gar nicht so schlimm, trotzdem pocht mein Herz schneller als sonst. Langsam füllt sich unsere Online-Gruppe via Zoom: 1, 2, 3, 4, 5, ... 8. Alle sind da. Noch einmal tief durchatmen und los geht es. Ich bitte meine Studienkolleginnen, eine angenehme Position einzunehmen, damit wir mit der Zentrierungsübung beginnen können.

„Atme einmal tief durch die Nase ein und durch den Mund wieder aus. Konzentriere dich nur auf deine Atmung ... Beim Ausatmen merkst du, wie die Muskelspannung in deinem Körper nachlässt. Konzentriere dich auf deinen Körper ... Wo spürst du noch Spannung? Schicke einen tiefen Atemzug an diese Stelle ... Wenn du die Augen wieder öffnest, schenke den anderen ein Lächeln. “

Die Achtsamkeitsübung dient dem Ankommen im Hier und Jetzt und unterstützt die Wahrnehmung der momentanen Gefühlslage. Danach folgt die erste Runde, in der alle Teilnehmenden drei Minuten zur Verfügung haben, um ihren Gefühlen und Gedanken Raum zu geben. Stille. Wird sich jemand melden? Soll ich warten? Soll ich anfangen? Ich warte ab. Dann meldet sich eine Kollegin. Ich bin erleichtert, stelle den Timer meines Handys auf drei Minuten ein und halte einen Gegenstand in die Kamera. In diesem Fall habe ich mich für ein Holzherz entschieden, mit dem ich allen signalisiere, wann die Zeit um ist. Es geht los, und die Kollegin beschreibt ihren Herzschag, ihre Atmung und wie es ihr heute geht.

Die drei Minuten sind um. Ich zeige das Holzherz in die Kamera. Stille. Ich frage nach, wer weitermachen möchte. So läuft das ab, bis jeder einmal an der Reihe war. Reden und Zuhören. Man kann, muss aber nicht reden. Man kann auch singen, tanzen, schreien oder die Stille aushalten. Wichtig ist nur, dass die vollen drei Minuten ausgeschöpft werden. Jede kann für sich entscheiden, was und wie sie es loswerden möchte.

Nachdem alle an der Reihe waren, folgt die zweite Runde. Ich stelle eine konkrete Frage, die stets positiv formuliert wird. Die erste Runde ist für alle frei, und oftmals wird hier über Sachen erzählt, die einen momentan belasten. Die zweite Runde soll dabei helfen, gestärkt aus der Gruppe zu gehen. Ich habe mehrere Fragen vorbereitet, da ich mich erst nach der ersten Runde je nach Stimmung der Teilnehmerinnen für eine Frage entscheiden wollte:

$\rightarrow$ Welche Aktivitäten machst du derzeit, die dir guttun?

$\rightarrow$ Wofür bist du dankbar?

$\rightarrow$ Worüber musstest du in letzter Zeit mal richtig laut lachen?

Ich entschied mich für die Frage nach wohltuenden Aktivitäten. Ohne lange zu warten, meldet sich die Erste: „Momentan tut es mir gut, wenn ich eine Runde im Wald spazieren gehe. Die Ruhe und der Duft der Natur geben mir Kraft."

Weiter geht's. Eine nach der anderen. Zeit stoppen. Zuhören. Holzherz zeigen. Abwarten, wer sich als Nächste bereit fühlt. Alle waren dran? Sehr gut. Ich schaue auf die Uhr. Eine Stunde ist vergangen.
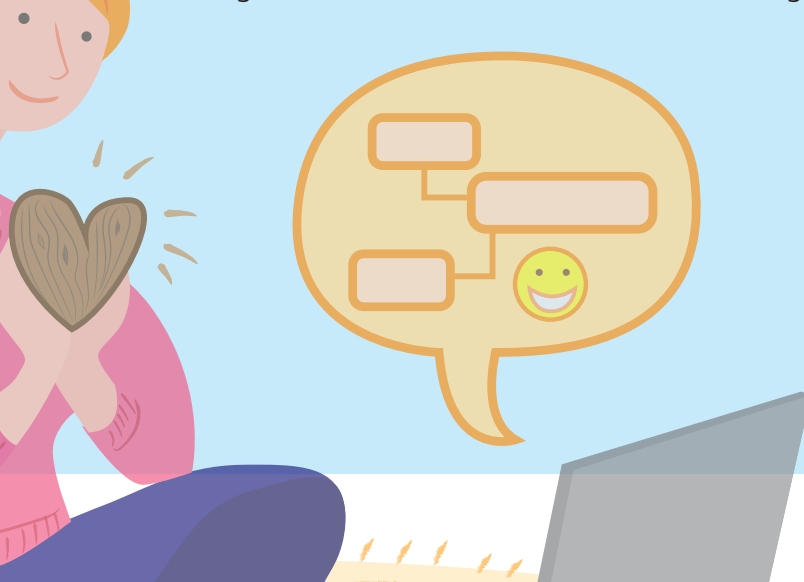

Unterbrechung des Alltags und der gewohnten Betätigungen [1]. Wesentlich am Konzept der Betätigungsunterbrechung ist, dass sie zeitlich begrenzt ist [5]. Aus einer ergotherapeutischen Perspektive formen Betätigungen die Identität einer Person. Betätigung und Identität stehen dabei in einer Wechselwirkung zueinander [6]. Durch eine Betätigungsunterbrechung wird nun diese Wechselwirkung und somit die Identität negativ beeinflusst [5]. Ebenfalls kann sich, so Nizzero et al. weiter, diese Occupational Disruption negativ auf soziale und/oder emotionale Aspekte des Seins auswirken. Ein plötzlicher Verlust von Betätigungen stehe im Zusammenhang mit einem Verlust von sozialen Netzwerken und sozialer Unterstützung, einem Verlust von Handlungsrollen und einem herabgesetzten Selbstwertgefühl.

Positive Auswirkungen von Aktivitäten $\rightarrow$ In Zeiten einer Betätigungsunterbrechung wirken folgende Arten von Aktivitäten positiv auf Resilienz und psychische Gesundheit [10, 11]:

$\rightarrow$ Aktivitäten, die soziale Verbundenheit fördern

$\rightarrow$ Aktivitäten, die dabei helfen, sich auf sich selbst zu fokussieren, wie etwa Meditation

$\rightarrow$ kreative Aktivitäten

$\rightarrow$ Aktivitäten, die ein größeres Bild des Seins in der Welt vermitteln

$\rightarrow$ Aktivitäten, die etwas zur Gemeinschaft beitragen

Lorrae Mynard (2020) beschreibt, dass es besonders in Krisenzeiten wichtig ist, bedeutungsvolle Betätigungen auszuführen und Strukturen und Routinen aufrechtzuerhalten, da diese Stabilität geben und mentale Gesundheit fördern. Das soziale Teilen von Emotionen ruft, ob positiv oder negativ, eine Reaktivierung der jeweiligen Emotion für die teilende Person hervor [7]. Durch das Teilen von positiven Emotionen werden diese bei der teilenden Person selbst verstärkt. Ob Emotionen online oder offline geteilt werden, mache dabei kaum einen Unterschied. Des Weiteren werden laut Rime et al. (2020) soziale Beziehungen durch den Austausch von Emotionen gestärkt, da das Gefühl der Verbundenheit steigt.

In einer Stellungnahme legt die American Occupational Therapy Association (AOTA) zu ergotherapeutischen Angeboten zur Förderung der psychischen Gesundheit und des Wohlbefindens fest: Aufgabe der Ergotherapie ist es, Menschen, die eine Unterbrechung in 
ihrer sozialen Partizipation erfahren, möglichst früh durch gesundheitsfördernde Programme zu unterstützen [8].

Während einer Occupational Disruption fördern Mindsharing-Gruppen die psychische Gesundheit durch Teilen und Zuhören, ohne moralisch zu bewerten. Die Teilnehmer/-innen können online in einer Zeit sozial partizipieren, in der die gewohnte Teilhabe größtenteils untersagt ist. Damit fördern MindsharingGruppen das Gefühl sozialer Verbundenheit. Sie helfen dabei, sich auf sich selbst zu fokussieren, ermöglichen ein Erleben von größeren Zusammenhängen der Welt und erlauben durch Zuhören und Teilen, etwas für die Gemeinschaft beizutragen. Darüber hinaus ermöglicht die Teilnahme an der Gruppe nicht nur ein Gefühl von sozialer Verbundenheit, sondern jede Einheit wird aufgrund der Struktur mit einem guten Gefühl beendet.

Ablauf einer Mindsharing-Gruppe $\rightarrow$ Diese Art des Teilens kann einmal oder regelmäßig stattfinden. In unserem Fall fand die Mindsharing-Gruppe zwei Mal wöchentlich über vier Wochen hinweg statt. Der Ablauf der Gruppe verlief immer nach demselben Aufbau. Die leitende Funktion der Gruppe wurde jedes Mal von einer anderen Teilnehmerin übernommen. Diese hatte die Aufgabe, alle Teilnehmenden durch die Stunde zu führen und auf diverse Rahmenbedingungen zu achten. Wie jede andere Teilnehmerin nahm auch sie aktiv an der gesamten Gruppe teil. Dadurch wurde vermittelt, dass wir alle gleichwertig sind und in diesem Setting keine Hierarchie herrscht.

Die Moderation startete stets mit einer Zentrierungsübung, die den Teilnehmenden helfen sollte, in Ruhe anzukommen (॰ BEISPIEL). In weiterer Folge hatten die Teilnehmenden jeweils drei Minuten zur Verfügung, die sie individuell und frei füllen konnten. Die Reihenfolge wurde vorher nicht festgelegt. Man konnte sich melden, wenn man sich dazu bereit fühlte. Nachdem die ersten drei Minuten von allen in Anspruch genommen wurden, folgte die zweite Runde. In dieser stellte die Moderatorin der Gruppe eine spezifische Frage, zu welcher sich wieder jede drei Minuten lang auf ihre Art und Weise äußerte.

Jeder gestaltet seine Teilnahme intuitiv $\rightarrow$ Die Teilnehmenden können ihre drei Minuten frei gestalten. Es gibt nur wenige Gruppen- regeln, diese aber sind für den Prozess des Gefühle-Teilens wesentlich. Für die OnlineGruppe ist es wichtig, dass sich die Teilnehmenden alleine in einem ruhigen Raum befinden, in dem sie ungestört sind, eine stabile Internetverbindung sowie eine funktionierende Kamera am Laptop haben. Wer die Moderation übernimmt, ist eine Art Zeitwächter/-in und stellt für alle den Wecker.

Die Essenz der Mindsharing-Gruppe ist das freie Sprechen (oder Nicht-Sprechen). Das heißt, das Gesagte sollte man sich nicht schon vorher überlegen. Ziel ist das Sprechen „aus dem Bauch heraus“, um das in unserer Gesellschaft tief verankerte analytische Kommunizieren zu vermeiden. Die drei Minuten dienen dem intuitiven Ausdruck der eigenen Gefühle durch Verbalisieren, Schweigen, Schreien, Tanzen oder Sonstiges. Für die Zuhörenden ist es wichtiger, die Sprechenden nicht zu unterbrechen und nicht zu analysieren, sondern bewusst mitfühlend zuzuhören.

In diesen drei Minuten wird nicht auf Aussagen anderer Teilnehmenden geantwortet und auch kein Feedback oder Ratschläge gegeben. Stattdessen konzentrieren sich alle allein auf das, was die Person mit ihnen teilen möchte, und auf das eigene Gefühl in diesem Moment.
Gefühl der Zugehörigkeit $\rightarrow$ Wie der Name Mindsharing schon sagt, schafft die Gruppe Raum für das Teilen von Gefühlen und Gedanken. Der Austausch führt dazu, dass man bemerkt, nicht allein in dieser Situation zu sein. Dadurch entsteht ein starkes Gefühl der Zugehörigkeit. Den Teilnehmenden wird bewusst, dass auch andere Personen im Laufe der Krise ähnliche Phasen durchleben wie sie selbst, obwohl die Lebensumstände der anderen sich von den eigenen unterscheiden. Wichtig dabei ist, dass alle Teilnehmenden vorab zustimmen, dass das Gesagte innerhalb der Gruppe bleibt.

Es wird akzeptiert, dass nicht immer alles gut ist und dass emotionale Zustände wechseln. Andere Personen teilen oftmals Gefühle, die einem selbst vertraut sind. Damit nicht allein zu sein, kann die innere Anspannung lösen. Durch das Teilen, wie andere mit der Situation umgehen, werden die Gruppenmitglieder inspiriert.

Gerade die zweite Runde regte dazu an, sich von den anderen Ideen zu holen, um in dieser ungewissen Zeit trotzdem produktiv sein zu können und sich selbst etwas Gutes zu tun. Im Allgemeinen wurde es als sehr befreiend empfunden, über die Dinge zu sprechen,

\section{Do}

Ich fühlte mich einsam und hatte Angst, dass es mir schlecht gehen wird in dieser Zeit. Die Mindsharing-Gruppe gab mir enorm viel Kraft. Ich konnte meine tiefsten Gedanken aussprechen, weinen und lachen, aber auch schweigen. Alles so, wie ich es gerade benötigte. Ich habe mich nicht nur von negativen Emotionen und Gedanken befreit, sondern habe auch gesehen, wie andere versuchen, das Beste für sich daraus zu machen. Das hat mich inspiriert, mir selbst Gutes zu tun und die Situation, wenn auch nur ein wenig, zu verbessern.

In der Mindsharing-Gruppe wurde ich angeregt, mir Gedanken über mich und bestimmte Fragen zu machen. Zum Beispiel, was Corona mit mir macht, was mir in dieser Zeit hilft, was mich zum Lachen bringt, wofür ich dankbar bin. Auch außerhalb der Gruppe reflektierte ich mehr als zuvor und übte mich in Akzeptanz gegenüber mir selbst und anderen. 
die einen im Moment beschäftigten, ohne dafür Tipps oder Ratschläge zu bekommen. Dadurch, dass auf das Gesagte nicht geantwortet wurde, außer es war explizit erwünscht, konnten auch die innersten Gedanken ausgesprochen werden. Es wurde spürbar, dass dies eine befreiende Wirkung hat.

Ein weiterer Benefit der Mindsharing-Gruppe ist, dass durch die beiden wöchentlichen Termine Orientierung und Struktur in einer Zeit geschaffen wird, in der ein Tag wie der andere ist. Ein zusätzlicher und durchaus bereichernder Aspekt der Gruppe war zu lernen, mitfühlend, geduldig und anteilnehmend zuzuhören. Von dieser Fähigkeit profitieren wir auch im alltäglichen und therapeutischen Umgang mit anderen. Das einfühlsame Anhören von Sorgen und Problemen, ohne dabei ungefragt Ratschläge oder Tipps zu geben, sorgt für eine angenehme Gesprächsbasis.

Ein neues und unkonventionelles Format $\rightarrow$ Aufgrund unserer positiven Erfahrungen mit der Mindsharing-Gruppe und dem Wissen, dass die Corona-Zeit auch für andere Studierende sehr belastend ist, war unser Ziel, dieses Angebot für alle Studierenden der Fachhochschule zugänglich zu machen. Dabei sammelten wir Erkenntnisse darüber, wie MindsharingGruppen implementiert werden können und was dabei herausfordernd sein kann - im Setting einer Fachhochschule. Wir sind aber überzeugt, dass sich diese Intervention auch für die Arbeit mit verschiedenen Klient(inn)engruppen eignet.

In Erwartung einer großen Resonanz versandten wir per E-Mail Einladungen für eine
Informationsrunde via Zoom. Allerdings nahm niemand das Angebot in Anspruch. Gründe für dieses scheinbare Desinteresse wurden gemeinsam reflektiert und bei Studierenden erfragt. Stolpersteine waren:

Das Format der Mindsharing-Gruppe ist neu und unkonventionell, weshalb vermutlich die Hemmschwelle für eine regelmäßige Teilnahme hoch war. Studierende, die zu dieser Problematik befragt wurden, gaben an, dass die Vorstellung, ihre Gefühle und Gedanken fremden Personen anzuvertrauen, sie skeptisch mache. Zudem bestand die Angst, dass Gesagtes in der Gruppe nach außen getragen wird. Ein weiterer Grund für die mangelnde Anteilnahme war, dass sich die Studierenden nicht für zwei wöchentliche Fixtermine binden wollten. Diese sind jedoch hinsichtlich einer nachhaltigen positiven Wirkung der Mindsharing-Gruppe zielführend. Zusätzlich wurden die Vorlesungen auf Online Plattformen verlegt, weshalb viele Studierende nicht noch mehr Zeit vor dem Bildschirm verbringen wollten.

Das legt die Vermutung nahe, dass sich viele Menschen insbesondere in stressigen Lebensphasen wenig Zeit für ihre psychische Gesundheit nehmen. Das klingt paradox und wäre interessant, näher zu untersuchen.

Eine Lösung wäre es, Gruppen anzusprechen, in denen sich die Teilnehmenden bereits kennen. Ebenso erwies es sich als effektiv, über Social Media und Mundpropaganda zu werben. Auf jeden Fall sollte die Vermittlung des Angebots persönlich erfolgen. Wichtig ist auch, dass wir im Falle eines weiteren Lockdowns wissen, dass der ideale Zeitpunkt eines Starts von Mindsharing-Gruppen zu Beginn der Krise ist. Erfreulicherweise kam schlussendlich doch eine weitere Gruppe zustande, die sehr gut funktionierte.

\section{Rüstzeug für eine Betä-} tigungsunterbrechung $\rightarrow$ Unsere Intention ist es, Ihnen Rüstzeug für eine Betätigungsunterbrechung zu liefern, die eine größere Gruppe von Menschen gleichzeitig betrifft. Aus unserer Sicht sind Mindsharing-Gruppen eine Interventionsform, von der Studierende, Klient(inn)engruppen in der Rehabilitation, in betreuten Wohngemeinschaften oder auch Angehörige profitieren könnten.

Georg Gappmayer, Marie-Sophie Band,

Carina M. King, Sophie Oswald, Tina Gruber, Ramona Sieberer, Carola Katzenbeisser, Sarah Brandl

Literaturverzeichnis

www.thieme-connect.de/products/ergopraxis $>$ „Ausgabe $11-12 / 20$ “

\section{Autoren}

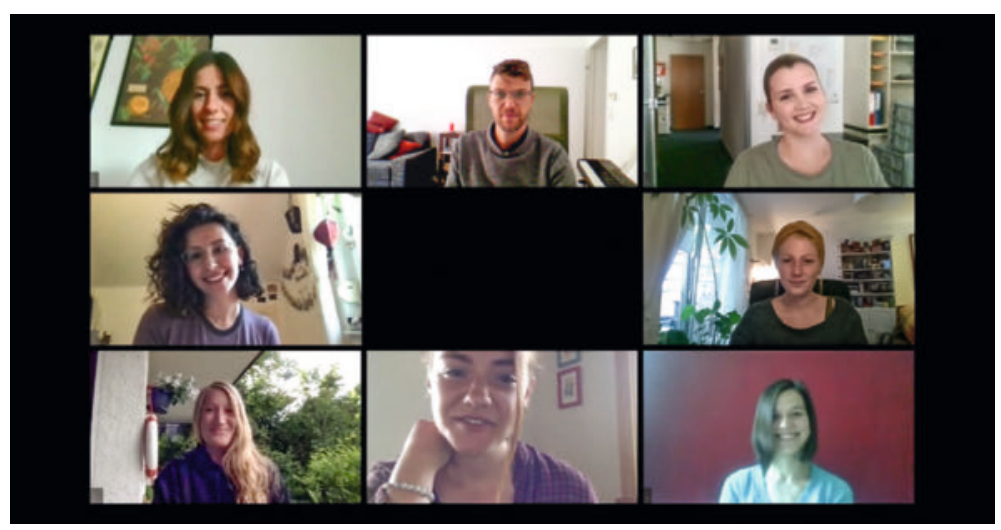

Georg Gappmayer, wissenschaftlicher Mitarbeiter im Studiengang Ergotherapie, Fachhochschule Wiener Neustadt, Österreich Marie-Sophie Band, Carina M. King, Sophie Oswald, Tina Gruber, Ramona Sieberer, Carola Katzenbeisser, Sarah Brandl, Absolventinnen des Studiengangs Ergotherapie der Fachhochschule Wiener Neustadt, Österreich

Wenn Sie mehr Information wünschen oder uns Ihre Erfahrungen mit diesem oder einem ähnlichen Gruppenformat mitteilen möchten, freuen wir uns über eine Kontaktaufnahme unter georg.gappmayer@fhwn.ac.at. 